\title{
Activation of Janus kinase 1 confers poor prognosis in patients with non-small cell lung cancer
}

\author{
DAN LIU ${ }^{1 *}$, YI HUANG ${ }^{2 *}, \mathrm{LI}_{\mathrm{ZHANG}}^{3}$, DONG-NI LIANG ${ }^{4}$ and $\mathrm{LI} \mathrm{LI}^{3}$ \\ ${ }^{1}$ Department of Respiratory Medicine, West China Hospital of Sichuan University, Chengdu, Sichuan 610041; \\ ${ }^{2}$ Clinical Laboratory Department, Sichuan Academy of Medical Sciences \& Sichuan Provincial People's Hospital, \\ Affiliated Hospital of The University of Electronic Science and Technology of China, Chengdu, Sichuan 610072; \\ ${ }^{3}$ State Key Laboratory of Biotherapy, West China Hospital of Sichuan University, Chengdu, Sichuan 610093; \\ ${ }^{4}$ Department of Pathology, Sichuan Academy of Medical Sciences \& Sichuan Provincial People's Hospital, Affiliated \\ Hospital of University of Electronic Science and Technology of China, Chengdu, Sichuan 610072, P.R. China
}

Received September 19, 2015; Accepted April 4, 2017

DOI: $10.3892 / \mathrm{ol} .2017 .6690$

\begin{abstract}
The activation of Janus kinase 1 (JAK1) has been reported to occur in non-small cell lung cancer (NSCLC), activating the JAK/signal transducers and activators of transcription cascade. However, the association between JAK1 activation and the prognostic value in NSCLC remains unclear. The present study initially investigated the association between expression of the activated form of JAK1 (p-JAK1) and prognosis in patients with NSCLC. A cohort of 142 resected primary NSCLC tissue samples, including 74 adenocarcinoma (ADCC) and 68 squamous cell carcinoma samples, were analyzed. p-JAK1 expression status was determined by immunohistochemistry. Evaluation of epidermal growth factor receptor (EGFR) gene amplification by fluorescence in situ hybridization was subsequently performed in 74 ADCC samples. The prognostic significance of p-JAK1 expression and EGFR gene amplification were evaluated with univariate and multivariate survival analyses. Compared with normal lung tissue, $\mathrm{p}-\mathrm{JAK} 1$ expression level was significantly increased in NSCLC $(\mathrm{P}<0.001)$. Positive p-JAK1 expression indicated a poor prognosis, particularly for patients in early stages (stage I/II, including tumor size $<3 \mathrm{~cm}$, Lymph node invasion $\mathrm{N} 0 / 1$; all $\mathrm{P}<0.05)$. p-JAK1 expression was an independent predictor of a poor prognosis $(\mathrm{P}=0.022)$. The overall
\end{abstract}

Correspondence to: $\mathrm{Dr} \mathrm{Yi}$ Huang, Clinical Laboratory Department, Sichuan Academy of Medical Sciences \& Sichuan Provincial People's Hospital, Affiliated Hospital of The University of Electronic Science and Technology of China, 32 West Section 2, 1 Ring Road, Chengdu, Sichuan 610072, P.R. China

E-mail: hwuangyi@foxmail.com

*Contributed equally

Key words: phosphorylated Janus kinase 1, non-small cell lung cancer, epidermal growth factor receptor, fluorescence in situ hybridization, prognosis survival time for patients with positive $\mathrm{p}-\mathrm{JAK} 1$ expression and EGFR-amplified tumors was significantly shortened compared with patients with tumors negative for one or both features (both features present vs. neither feature present, $\mathrm{P}<0.001$ ). The results provided clinical evidence that the activation of JAK1 was an independent prognostic factor, particularly in early stage NSCLC. The combination of EGFR gene amplification and p-JAK1 expression may be a novel target for the selection of individual therapy strategies and predicting the effects of therapy for NSCLC.

\section{Introduction}

Lung cancer is one of the leading causes of cancer-associated mortality, accounting for $27 \%$ (including $26 \%$ for females, and $28 \%$ for males), and non-small cell lung cancer (NSCLC) accounts for $80-85 \%$ of all lung cancer-associated mortalities $(1,2)$. Despite advances in the understanding of the molecular mechanisms of lung cancer and the development of novel chemotherapeutic agents, the 5-year survival rates for lung and bronchus cancer remained $<18 \%$ from 2004 to 2010 (2). A number of studies have focused on progressing the understanding of oncogenic kinase signaling pathways, which has provided targets for developing effective therapeutic strategies in order to improve clinical outcomes (3).

One of the potential candidates for therapy is the Janus kinase/signal transducers and activators of transcription (JAK/STAT) pathway. JAK/STAT is one of the pleiotropic cascades that may transduce a multitude of signals for development and homeostasis in animals, from humans to flies (4). JAK family members have been reported to be dysregulated in malignant tumors, including in colorectal, prostate and myeloproliferative cancer (5-8). The mammalian JAK family includes four members: JAK1, JAK2, JAK3 and Tyk2. All JAKs exhibit broad patterns of expression with the exception of JAK3, which is restricted to leukocytes (9).

JAK1 binds to various cytokines non-covalently to mediate cell proliferation and differentiation (10). JAK1 knockout mice die perinatally (9). JAK1 mutation has been reported in hepatocellular carcinoma, acute lymphoblastic 
leukemia, lung and gastric cancer $(10,11)$. Phosphorylated (p)-JAK1, the active form of JAK1, mediates the phosphorylation of receptors and the major substrates for JAK family members, STATs (4). For example, p-JAK1 expression was detected in primary esophageal squamous cell carcinoma and not in normal esophageal squamous cells. p-JAK1 expression was associated with a reduced overall survival time (12). Additionally, a previous study by the present authors demonstrated that JAK1 expression was significantly increased in NSCLC clinical samples compared with normal samples ( $\mathrm{P}>0.001)$, while p-JAK1 (Tyk 1022) showed trends of positive expression, though these did not reach statistical significance $(\mathrm{P}=0.055)$, potentially owing to a small sample size (13). This indicated that JAK1 activation was abnormal in NSCLC.

Lung adenocarcinoma (ADCC) is the most common histological subtype of NSCLC. Patients with ADCC and a high epidermal growth factor receptor (EGFR) copy number can be treated with EGFR-tyrosine kinase inhibitors $(14,15)$. EGFR gene amplification has also been reported to be associated with prognosis of lung cancer, though there is some controversy in this regard $(16,17)$. However, the combination of EGFR gene amplification and JAK1 activation for predicting cancer prognosis has not been extensively studied. This has incited the present study, which will investigate associations between JAK1 activation, EGFR gene amplification and survival status in patients with NSCLC.

\section{Materials and methods}

Tissue collection. The study cohort consisted of 142 patients (40 female and 102 male) with a median age of 63 years (range, 20-84 years). A total of 142 paraffin-embedded resected primary NSCLC samples were analyzed from the archives of the Pathology Department at Sichuan Provincial People's Hospital (Chengdu, China) from December 2004 to February 2007, including 74 cases of ADCC and 68 cases of squamous cell carcinoma (SqCC) A total of 142 adjacent normal pulmonary tissue specimens were also resected in the same tissue blocks. Staging was performed according to the International Union Against Cancer's tumor-node-metastasis system (18). Differentiation and histological type were scored according to the World Health Organization classification for NSCLC (19). None of the patients had received neoadjuvant therapy prior to surgical resection. Following the surgery, the patients underwent standard therapy procedure, according to the National Comprehensive Cancer Network Clinical Practice Guideline for Oncology, NSCLC, 2004 (20). Informed consent was obtained from all individuals included in the present study. Institutional review board approval for the study was obtained from Sichuan Provincial People's Hospital.

Immunohistochemical staining (IHC). IHC staining was performed as previously described (3). Briefly, the 4- $\mu \mathrm{m}$ sections underwent deparaffinization, hydration and endogenous peroxide blocking. Antigen retrieval was performed by heating at $95^{\circ} \mathrm{C}$ for $30 \mathrm{~min}$ in Tris/ethylenediaminetetraacetic acid retrieval solution. The sections were blocked with $3 \%$ bovine serum albumin (Sigma-Aldrich; Merck KGaA,
Table I. p-JAK1 expression in NSCLC and adjacent normal tissues.

\begin{tabular}{lcrr}
\hline Parameter & $\begin{array}{c}\text { p-JAK1 } \\
\mathrm{n}(\%)\end{array}$ & $\begin{array}{c}\text { p-JAK1 } \\
\mathrm{n}(\%)\end{array}$ & P-value $^{\mathrm{a}}$ \\
\hline NSCLC tissue & $56(39.4)$ & $86(60.6)$ & $<0.001$ \\
Normal control & $11(7.7)$ & $131(92.3)$ & \\
\hline
\end{tabular}

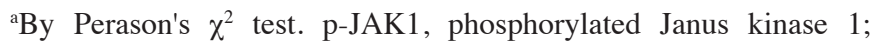
NSCLC, non-small cell lung carcinoma.

Darmstadt, Germany) and incubated with primary p-JAK1 antibody (cat. no. 11149, 1:100 dilution; Signalway Antibody LLC., College Park, MY, ML, USA) overnight at $4^{\circ} \mathrm{C}$. The membranes were subsequently incubated with enough secondary peroxidase-labeled polymer-conjugated goat anti-rabbit antibody to cover the specimen (cat. no. K4007; undiluted; EnVsion; Dako; Agilent Technologies, Inc., Santa Clara, CA, USA), according to the manufacturer's protocol, for $30 \mathrm{~min}$ at room temperature. For staining, 3,3-daiminobenzidine chromogen was applied. Finally, the sections were counterstained with hematoxylin for $3 \mathrm{~min}$ at room temperature, fixed and mounted.

Scoring for immunohistochemical staining. Scoring for immunochemical staining was evaluated as previously described (3) by two independent pathologists. Briefly, the expression of p-JAK1 was assessed semi-quantitatively on the basis of criteria that accounted for the fraction and intensity of immunostaining of the tumor cells. The fraction score was defined as: $0(0 \%), 1(<20 \%), 2(20-50 \%)$ and $3(>50 \%)$. The intensity of p-JAK1 staining was scored as 0 (no appreciable staining), 1 (barely detectable staining), 2 (readily identifiable brown staining) and 3 (dark brown staining). A total score was calculated by multiplying the fraction and the intensity score. A tumor sample was considered positive if the score was $\geq 4$, and negative otherwise.

Determination of EGFR gene amplification by fluorescence in-situ hybridization (FISH). EGFR FISH analysis was performed with the dual-color EGFR SpectrumOrange/CEP7 SpectrumGreen probe and paraffin pretreatment reagent kit (both from Vysis; cat. nos. 05J48-001 and 32-801210, respectively; Abbott Laboratories, Chicago, IL, USA) as previously described (21). Briefly, the paraffin sections were deparaffinized, dehydrated and digested with protease $\mathrm{K}$. The slides were denatured by heating to $75^{\circ} \mathrm{C}$ for $5 \mathrm{~min}$ and dehydrated in ethanol. The probes were denatured for $5 \mathrm{~min}$ at $75^{\circ} \mathrm{C}$ prior to hybridization. Each slide was hybridized at $37^{\circ} \mathrm{C}$ overnight and washed in $2 \mathrm{X}$ saline-sodium citrate buffer/0.3\% NP40 at $72^{\circ} \mathrm{C}$ for $2 \mathrm{~min}$. The nuclei were counterstained with DAPI/antifade 1 (Vysis; Abbott Laboratories, Chicago, IL, USA). A minimum of 100 non-overlapping tumor cell nuclei were scored in each case, according to the University of Colorado Cancer Center criteria (22). The BX-51/Genus FISH Imaging system (DP71/70/DP30BW software, DP-BSW Ver.03.02) was used for imaging (Olympus, Tokyo, Japan). 
Table II. Clinical characteristics and prognosis of patients with non-small cell lung carcinoma.

\begin{tabular}{|c|c|c|c|}
\hline Parameter & Patients, $\mathrm{n}$ & Median survival time, range (months) & P-value \\
\hline Age & & & 0.005 \\
\hline$<60$ years & 56 & $63(3-94)$ & \\
\hline$\geq 60$ years & 86 & $60(1-96)$ & \\
\hline Gender & & & 0.561 \\
\hline Female & 40 & $63(3-96)$ & \\
\hline Male & 102 & $61(1-94)$ & \\
\hline Histological type & & & 0.959 \\
\hline Adenocarcinoma & 74 & $61(3-96)$ & \\
\hline Squamous cell carcinoma & 68 & $62.5(1-94)$ & \\
\hline Tumor size & & & 0.446 \\
\hline$<3 \mathrm{~cm}$ & 59 & $61.5(6-96)$ & \\
\hline$\geq 3 \mathrm{~cm}$ & 83 & $60(1-94)$ & \\
\hline Lymph node invasion & & & $<0.001$ \\
\hline $\mathrm{N} 0 / 1$ & 122 & $63(1-96)$ & \\
\hline $\mathrm{N} 2 / 3$ & 20 & $39(3-67)$ & \\
\hline Distant metastasis & & & 0.973 \\
\hline M0 & 138 & $61(1-96)$ & \\
\hline M1 & 4 & $57.5(41-85)$ & \\
\hline TNM stage & & & 0.001 \\
\hline $\mathrm{I} / \mathrm{II}$ & 92 & $62.5(1-96)$ & \\
\hline III/IV & 50 & $51.5(3-89)$ & \\
\hline
\end{tabular}

${ }^{a}$ By log-rank test. TNM, tumor-node-metastasis staging system.

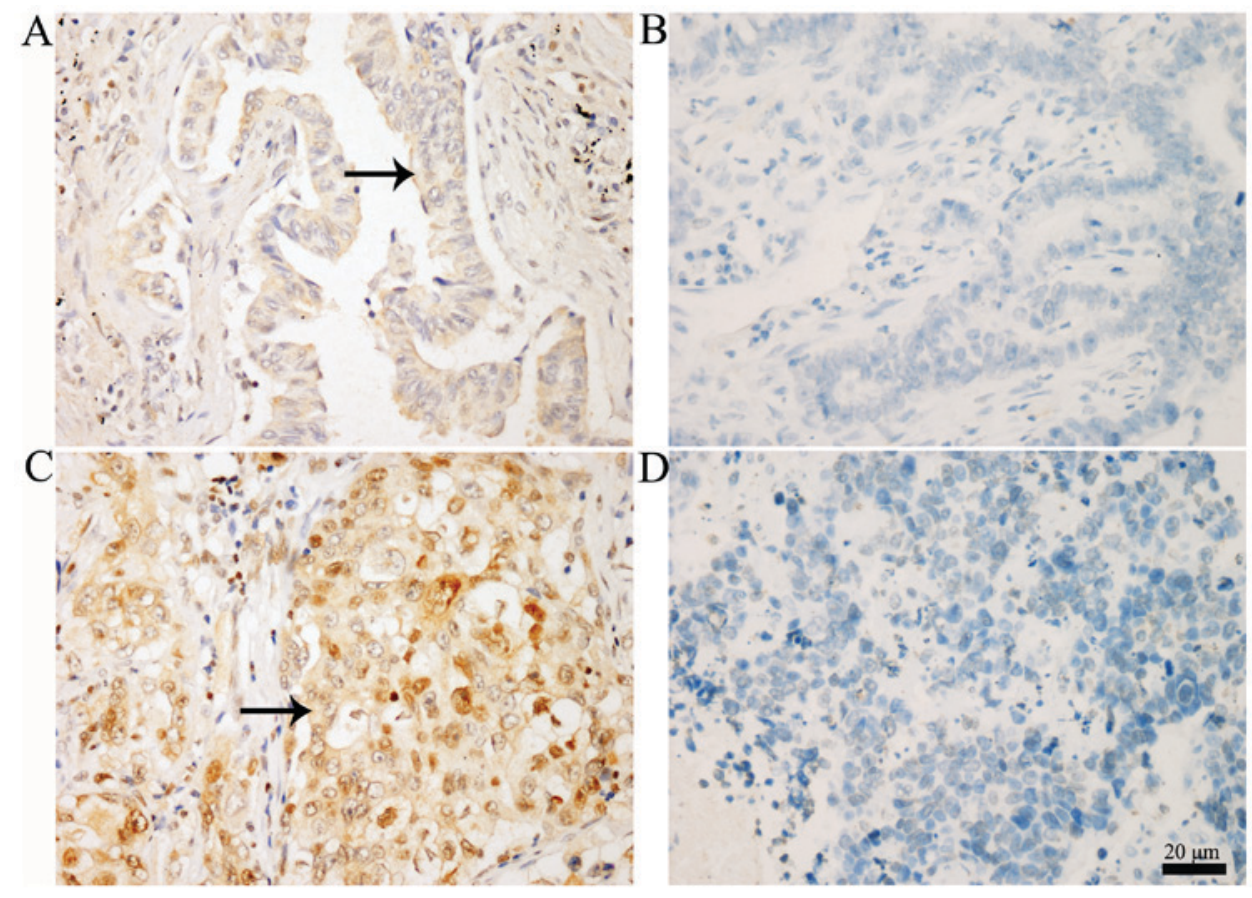

Figure 1. Representative images of p-JAK1 positive and negative expression in non-small cell lung carcinoma. Expression of p-JAK1 was observed in the cytoplasm and nucleus, as indicated by the arrows. (A) Positive and (B) negative p-JAK1 expression in adenocarcinoma; (C) positive and (D) negative p-JAK1 expression in squamous cell carcinoma. Magnification, x400. p-JAK1, phosphorylated Janus kinase 1.

Statistical analysis. The association between clinical characteristics and p-JAK1 expression was determined using Pearson's $\chi^{2}$ test. When one of the expected values in a $2 \times 2$ table was $<5$, Fisher's exact test would be used. The 
Table III. p-JAK1 expression and clinical characteristics in patients with non-small cell lung cancer.

\begin{tabular}{|c|c|c|c|}
\hline Characteristic & p-JAK $1^{+}$ & p-JAK $1^{-}$ & P-value \\
\hline Age & & & $0.867^{\mathrm{a}}$ \\
\hline$<60$ & 22 & 35 & \\
\hline$\geq 60$ & 34 & 51 & \\
\hline Gender & & & $0.290^{\mathrm{a}}$ \\
\hline Female & 13 & 27 & \\
\hline Male & 43 & 59 & \\
\hline Histological type & & & $0.014^{\mathrm{a}}$ \\
\hline Adenocarcinoma & 22 & 52 & \\
\hline Squamous cell carcinoma & 34 & 34 & \\
\hline Tumor size & & & $0.029^{\mathrm{a}}$ \\
\hline$<3 \mathrm{~cm}$ & 17 & 42 & \\
\hline$\geq 3 \mathrm{~cm}$ & 39 & 44 & \\
\hline Lymph node invasion & & & $0.297^{\mathrm{a}}$ \\
\hline $\mathrm{N} 0 / 1$ & 46 & 76 & \\
\hline $\mathrm{N} 2 / 3$ & 10 & 10 & \\
\hline Distant metastasis & & & $0.300^{\mathrm{b}}$ \\
\hline M0 & 53 & 85 & \\
\hline M1 & 3 & 1 & \\
\hline TNM stage & & & $0.009^{\mathrm{a}}$ \\
\hline $\mathrm{I} / \mathrm{II}$ & 29 & 63 & \\
\hline III/IV & 27 & 23 & \\
\hline
\end{tabular}

'By Perason's $\chi^{2}$ test; by Fisher's exact test. p-JAK1, phosphorylated Janus kinase 1; TNM, tumor-node-metastasis staging system.

Table IV. Survival distributions between groups separated by clinical characteristics and p-JAK1 expression.

\begin{tabular}{|c|c|c|c|}
\hline Comparisons & Hazard ratio & Confidence interval $(95 \%)$ & P-value ${ }^{a}$ \\
\hline \multicolumn{4}{|l|}{ NSCLC } \\
\hline p-JAK $1^{+}$vs. p-JAK $1^{-}$ & 1.929 & $1.100-3.382$ & 0.022 \\
\hline $\mathrm{N} 0 / 1$ vs. $\mathrm{N} 2 / 3^{\mathrm{b}}$ & 4.256 & $2.253-8.037$ & $<0.001$ \\
\hline \multicolumn{4}{|l|}{ ADCC } \\
\hline p-JAK1+ vs. p-JAK $1^{-}$ & 2.285 & $1.014-5.147$ & 0.046 \\
\hline TNM stage I/II vs. III/IV & 3.551 & $1.573-8.017$ & 0.002 \\
\hline \multicolumn{4}{|l|}{$\mathrm{SqCC}$} \\
\hline $\mathrm{N} 0 / 1$ vs. N2/3 & 4.110 & $1.481-11.407$ & 0.007 \\
\hline
\end{tabular}

${ }^{a}$ By Cox regression multivariate analysis; ${ }^{b}$ Lymph node invasion. ADCC, adenocarcinoma; NSCLC, non-small cell lung carcinoma; p-JAK1, phosphorylated Janus kinase 1; TNM, tumor-node-metastasis staging system; SqCC, squamous cell carcinoma.

Kaplan-Meier method was used for the univariate analysis of survival time between groups based on clinical characteristics and p-JAK1 expression. Multivariate analysis was performed using Cox regression model analysis. Only markers that were significant predictors in univariate analysis were included in the multivariate analysis. All tests were two-sided and $\mathrm{P}<0.05$ was considered to indicate a statistically significant difference. SPSS 17.0 for Windows (SPSS Inc., Chicago, IL, USA) was used for these analyses.

\section{Results}

JAK1 expression in non-small cell lung cancer tissues. Representative IHC staining images of p-JAK1 expression in NSCLC and adjacent normal lung tissues are displayed in Fig. 1. Positive p-JAK1 expression was observed in the cytoplasm and nucleus, as indicated by arrows. In the NSCLC tissues, 56/142 cases (39.4\%) exhibited positive p-JAK1 expression, whereas $11 / 142$ cases $(7.7 \%)$ were positive in the 

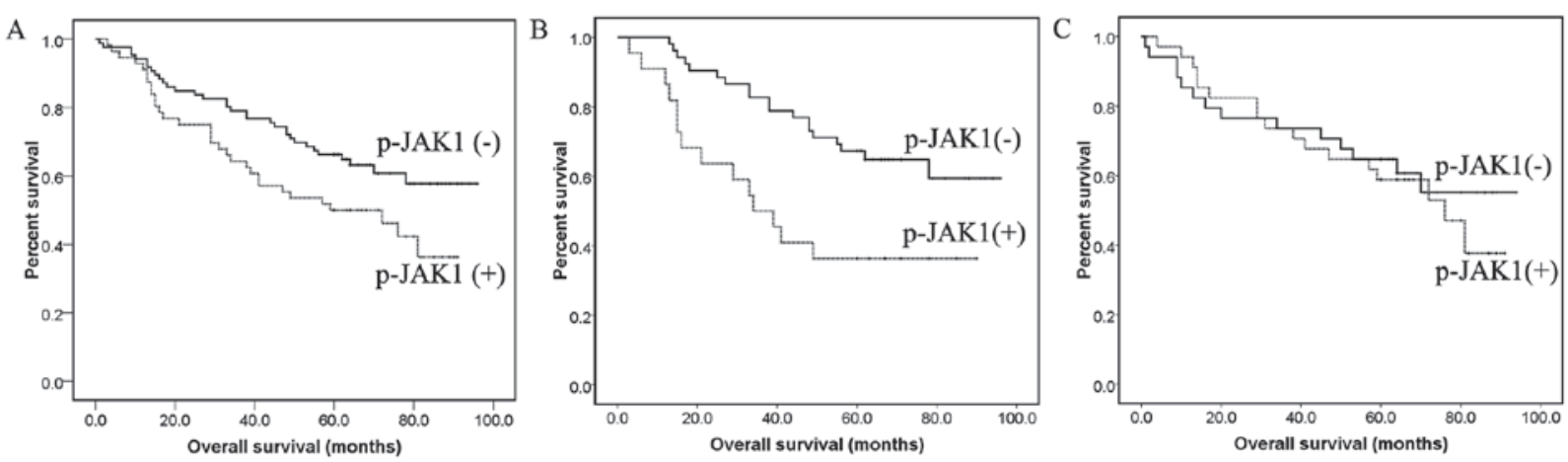

Figure 2. Association between p-JAK1 expression status and survival time in NSCLC. Kaplan-Meier curves compare the overall survival time of patients with positive (dotted line) or negative (solid line) p-JAK1 expression. Kaplan-Meier curves for (A) all types of NSCLC, (B) adenocarcinoma and (C) squamous cell carcinoma subtypes are illustrated. p-JAK1, phosphorylated Janus kinase 1; NSCLC, non-small cell lung carcinoma.
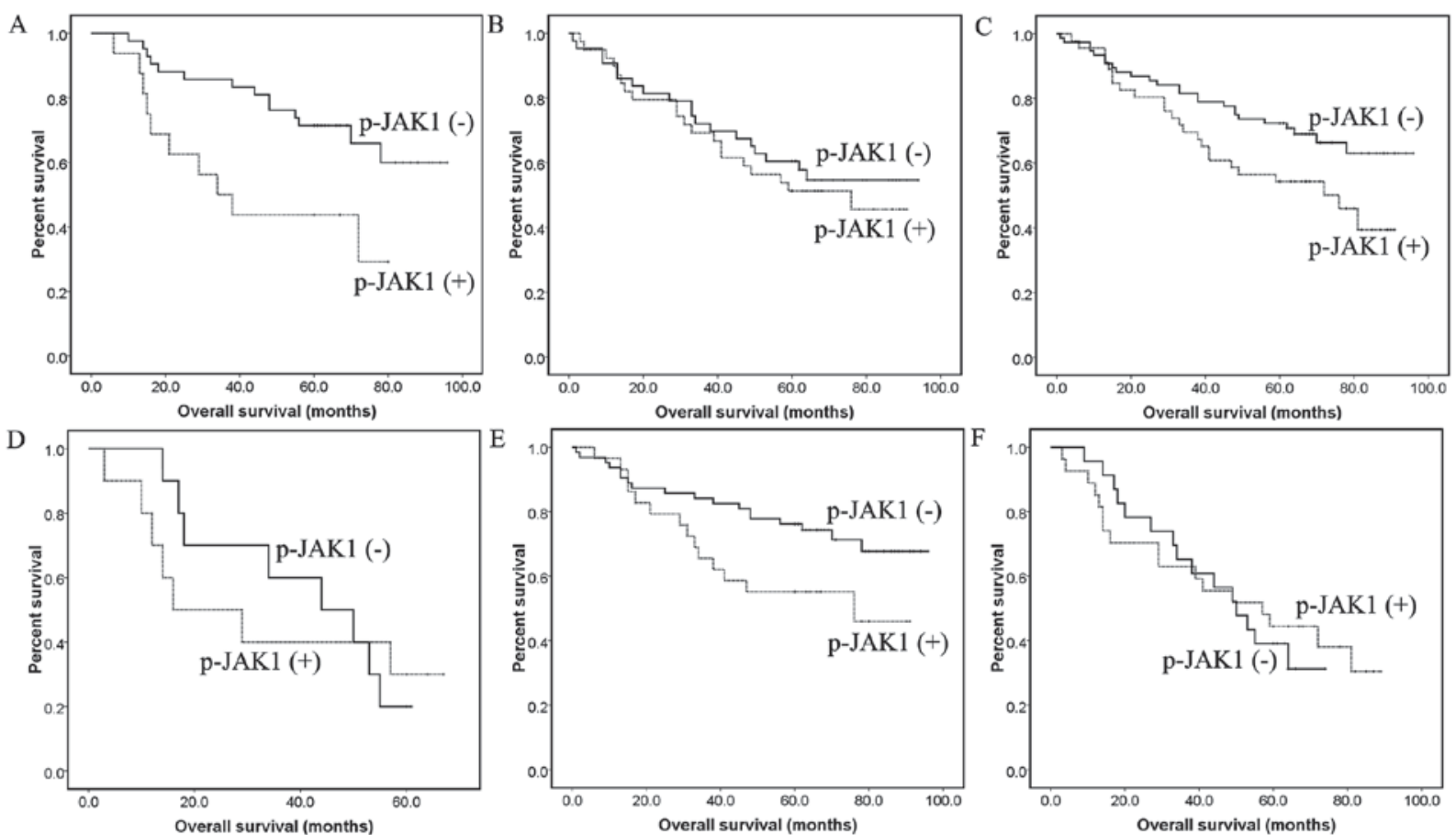

Figure 3. Association between p-JAK1 expression status and survival time in early and late stage NSCLC. Kaplan-Meier curves for patient survival are stratified by p-JAK1 positive (dotted line) and negative expression (solid line) in (A) Tumor size $<3 \mathrm{~cm}$ (B) Tumor size $\geq 3 \mathrm{~cm}$, (C) N0/1 (D) N2/3 (E) stage I/II and (F) stage III/IV groups, respectively. p-JAK1, phosphorylated Janus kinase 1; NSCLC, non-small cell lung carcinoma.

adjacent normal tissues (Table I). The difference was highly significant $(\mathrm{P}<0.001)$.

Association between clinical characteristics and prognosis in patients with NSCLC. As listed in Table II, follow-up information was available for all patients. Survival time differed significantly by age ( $>60$ vs. $\leq 60)$, lymph node invasion ( $\mathrm{N} 0 / 1$ vs. N2/3) and stage (I/II vs. III/IV; $\mathrm{P}=0.005, \mathrm{P}<0.001$ and $\mathrm{P}=0.001$, respectively). Gender, tumor size and histological type were not significantly associated with survival time.

Association between clinical characteristics and p-JAKI expression. Pearson's $\chi^{2}$ or Fisher's exact test were performed to assess the significance of associations between clinical characteristics and p-JAK1 expression. As shown in Table III, p-JAK1 expression was associated with histological type, as $29.7 \%$ of ADCC samples and $50.0 \%$ of SqCC samples were $\mathrm{p}-\mathrm{JAK} 1^{+}(\mathrm{P}=0.014)$. $\mathrm{p}-\mathrm{JAK} 1$ expression status was also associated with tumor size and stage $(\mathrm{P}=0.021$ and $\mathrm{P}=0.009$, respectively). Other clinical characteristics, including age, gender, lymph node invasion and distant metastasis, were not associated with p-JAK1 expression status ( $\mathrm{P}>0.05)$.

Survival status and p-JAK1 expression. Positive p-JAK1 expression was associated with a poor prognosis in NSCLC ( $\mathrm{P}=0.039$; Fig. 2A-C). However when divided by histological type, the difference was only statistically significant in ADCC samples $(\mathrm{P}=0.007)$ and not in $\mathrm{SqCC}(\mathrm{P}=0.612)$. Subjects with p-JAK1 expression demonstrated reduced survival time in early stage NSCLC, including patients with tumor size $<3 \mathrm{~cm}$, $\mathrm{N} 0 / 1$ and stage $\mathrm{I} / \mathrm{II}(\mathrm{P}=0.016, \mathrm{P}=0.034$ and $\mathrm{P}=0.048$, respectively; Fig. 3A-F). The difference in survival time in patients 
Table V. Effects of p-JAK1 expression and EGFR gene duplication status on the prognosis of patients with lung adenocarcinoma, as determined by Kaplan-Meier analysis.

\begin{tabular}{lccr}
\hline Status & Patients, $n$ & Median survival time (months) & P-value $^{\text {a }}$ \\
\hline p-JAK1 $^{+} /$EGFR $^{+}$ & 7 & $16(3-60)$ & $<0.001$ \\
p-JAK1 $^{-} /$EGFR $^{-}$ & 30 & $61.5(15-96)$ & 0.004 \\
p-JAK1 $^{-}$EGFR $^{+}$ & 22 & $62(13-94)$ & 0.052 \\
p-JAK1 $^{+} /$EGFR $^{-}$ & 15 & $49(12-90)$ & \\
\hline
\end{tabular}

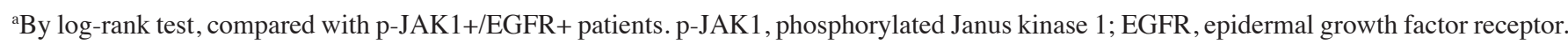

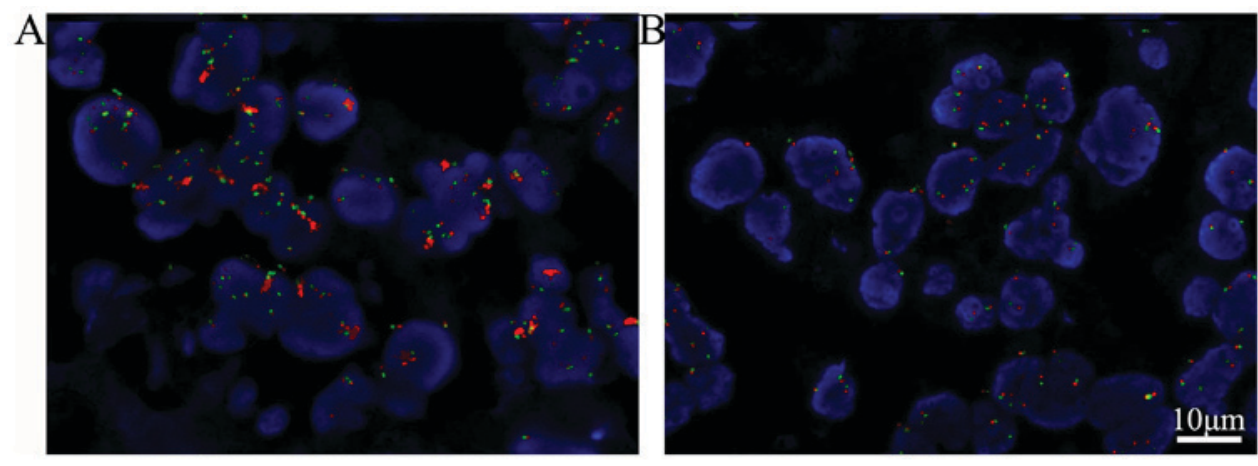

Figure 4. Determination of EGFR gene amplification by FISH. Evaluation of EGFR gene copy number by FISH analysis was performed using an EGFR (orange)/CEP 7 (green) probe with DAPI nuclear counterstaining. Representative examples are included of (A) a sample featuring EGFR gene duplication and (B) a sample with a normal number of EGFR genes. Magnification, x400. EGFR, epidermal growth factor receptor; FISH, fluorescence in situ hybridization.

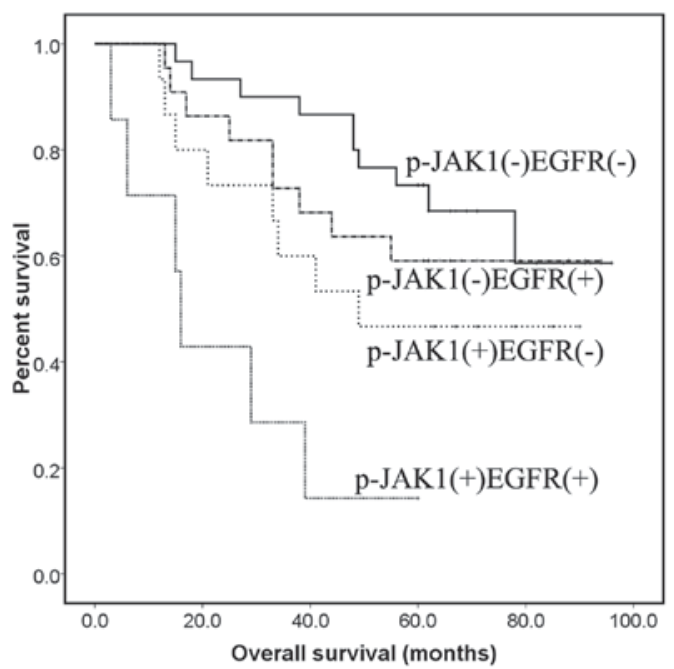

Figure 5. Association between the combination of p-JAK1 expression and EGFR gene duplication, and survival time in patients with lung adenocarcinoma. Kaplan-Meier curves for patient survival are stratified by p-JAK1 positive or negative expression with EGFR duplication positive or negative status. Solid line, p-JAK1//EGFR expression; dashed line, p-JAK1/EGFR half-dotted line, p-JAK1 ${ }^{+} / \mathrm{EGFR}^{-}$, dotted line, $\mathrm{p}-\mathrm{JAK} 1^{+} / \mathrm{EGFR}^{+}$. p-JAK1, phosphorylated Janus kinase 1; EGFR, epidermal growth factor receptor.

with late stage disease, including tumor size $\geq 3 \mathrm{~cm}, \mathrm{~N} 2 / 3$ or stage III/IV was not significant.

Survival status and p-JAK1 and EGFR expression in ADCC. To investigate whether associations between JAK1 activation,
EGFR gene amplification and prognosis in patients with ADCC were significant, EGFR status was examined with FISH analysis, and univariate survival analyses were performed for the 74 ADCC subjects (Table V). EGFR gene amplification was determined by FISH (Fig. 4), 29/74 ADCC cases exhibited EGFR gene amplification (designated as $\mathrm{EGFR}^{+}$). As shown in Table V, survival time for patients with p-JAK1 ${ }^{+} / \mathrm{EGFR}^{+}$was significantly reduced compared with those with $\mathrm{p}-\mathrm{JAK} \mathrm{1}^{-} / \mathrm{EGFR}-$ or $\mathrm{p}-\mathrm{JAK} 1^{-} / \mathrm{EGFR}^{+}$combinations $(\mathrm{P}<0.001$ and $\mathrm{P}=0.004$, respectively), but not the $\mathrm{p}-\mathrm{JAK} 1^{+} / \mathrm{EGFR}^{-}$combination $(\mathrm{P}=0.052$; Fig. 5). The results indicated that the EGFR amplification and $\mathrm{p}-\mathrm{JAK} 1^{+}$combination may be a novel target to inform the selection of individual therapy strategies and for predicting the effect of therapy in ADCC.

\section{Discussion}

Phosphorylation of JAK1 is important for the activation of JAK/STAT pathways and their downstream cascades (10). In the present study, it was identified that the activation of JAK1 was associated with a poorer prognosis in NSCLC $(\mathrm{P}=0.039)$, particularly in ADCC $(\mathrm{P}=0.007)$. p-JAK1 was identified as an independent predictor for poor prognosis $(\mathrm{P}=0.022)$. Overall survival time for patients with p-JAK $1^{+}$and EGFR gene duplication was significantly reduced compared with patients with one or neither trait $(\mathrm{P}=0.001)$.

A previous study by the present authors and studies by others have indicated that inhibition of JAK signaling exhibits anticancer and anti-angiogenetic effects in human cancer 
lines and xenograft tumors, including lung cancer (13,23-25). These results support the hypothesis that JAK1 activation is important for tumorigenesis in NSCLC. In the present study, when subdivided by histotype, differences in prognosis were identified as statistically significant. Positive p-JAK1 was an independent predictor for decreased survival times only for patients with ADCC, not SqCC. This finding suggests a specific role for JAK1 in lung ADCC. In several lung ADCC cell lines, JAK family inhibitor AZD1480 has demonstrated to be able to potently block STAT3 signaling and oncogenesis (24), which supports the results of the present study to some extent. However, the research on the effect of p-JAK1 expression on prognosis in lung SqCC has been limited. You et al (12) reported that JAK/STAT pathway activations were associated with shorter survival time in esophageal SqCC cases. As only 68 cases of SqCC patients were recruited to the present study, further evaluation should be performed using a larger amount of cases.

In the present study, it was further identified that tumor size $<3 \mathrm{~cm}, \mathrm{~N} 0 / 1$ and stage I/II patients with positive $\mathrm{p}-\mathrm{JAK} 1$ expression had shorter survival time (all $\mathrm{P}<0.05$ ), whereas the difference was not significant for patients in advanced stages (Fig. 3). This finding indicated that the activation of JAK1 may be an early event in the tumorigenesis of NSCLC. Therefore, p-JAK1 could be a target for early diagnosis and prognosis prediction.

In the present study, patients with $\mathrm{p}-\mathrm{JAK} 1^{+}$and EGFR duplication exhibited the poorest prognosis while patients with p-JAK ${ }^{-}$and no EGFR duplication exhibited the most favorable prognosis (median survival time, 16 vs. 61.5 months). Although contradictory results were previously reported (14,15), EGFR FISH status alone was not associated with the patient survival times (data not shown). To date, few studies have focused on the interaction between EGFR and JAKs at the cellular and molecular level. However, it has been identified that crosstalk between the JAK/STAT and EGFR pathways mediates adenomatous polyposis coil 1-driven intestinal stem cell hyperplasia in drosophila (26). Furthermore, prolactin, a JAK2-coupled cytokine receptor, has also been demonstrated to synergistically augment EGF signaling in T47D breast cancer cells (27). In NSCLC, how EGFR and JAK1 interact to exert biological effects should be further elucidated.

The present study indicated that JAK1 activation is associated with a poor prognosis in NSCLC, particularly in lung ADCC. p-JAK1 is an independent poor prognostic factor and a potential target for early diagnosis. The combination of EGFR gene amplification and JAK1 activation may be a novel tool for prognosis prediction in patients with lung ADCC.

\section{Acknowledgements}

The authors of the present study are grateful to the pathologist from the Department of Pathology, Sichuan Provincial People's Hospital for assisting in the collection of the tissue samples. The present study was supported by grants from the National Natural Foundation of China (grant no. 81201851), Support Program of Science \& Technology Department of Sichuan Province (grant no. 2014SZ0231) and Doctoral Foundation of Sichuan Provincial People's Hospital (grant no. 30305030563).

\section{References}

1. Peters S, Adjei AA, Gridelli C, Reck M, Kerr K and Felip E; ESMO Guidelines Working Group: Metastatic non-small-cell lung cancer (NSCLC): ESMO clinical practice guidelines for diagnosis, treatment and follow-up. Ann Oncol 23 (Suppl 7): vii56-vii64, 2012.

2. Siegel RL, Miller KD and Jemal A: Cancer statistics, 2015. CA Cancer J Clin 65: 5-29, 2015.

3. Liu D, Huang Y, Chen B, Zeng J, Guo N, Zhang S, Liu L, Xu H, Mo X and Li W: Activation of mammalian target of rapamycin pathway confers adverse outcome in nonsmall cell lung carcinoma. Cancer 117: 3763-3773, 2011.

4. Rawlings JS, Rosler KM and Harrison DA: The JAK/STAT signaling pathway. J Cell Sci 117: 1281-1283, 2004.

5. Constantinescu SN, Leroy E, Gryshkova V, Pecquet C and Dusa A: Activating Janus kinase pseudokinase domain mutations in myeloproliferative and other blood cancers. Biochem Soc Trans 41: 1048-1054, 2013.

6. Bergmann AK, Schneppenheim S, Seifert M, Betts MJ, Haake A. Lopez C, Maria Murga Penas E, Vater I, Jayne S, Dyer MJ, et al: Recurrent mutation of JAK3 in T-cell prolymphocytic leukemia. Genes Chromosomes Cancer 53: 309-316, 2014.

7. Wang SW and Sun YM: The IL-6/JAK/STAT3 pathway: Potential therapeutic strategies in treating colorectal cancer (Review) Int J Oncol 44: 1032-1040, 2014.

8. Gurbuz V, Konac E, Varol N, Yilmaz A, Gurocak S, Menevse S and Sozen S: Effects of AG490 and S3I-201 on regulation of the JAK/STAT3 signaling pathway in relation to angiogenesis in TRAIL-resistant prostate cancer cells. Oncol Lett 7: 755-763, 2014.

9. Schindler C, Levy DE and Decker T: JAK-STAT signaling: From interferons to cytokines. J Biol Chem 282: 20059-20063, 2007.

10. Xie H, Bae H, Noh J, Eun JW, Kim JK, Jung KH, Ryu JC, Ahn YM, Kim SY, Lee SH, et al: Mutational analysis of JAK1 gene in human hepatocellular carcinoma. Neoplasma 56: 136-140, 2009.

11. Jeong EG, Kim MS, Nam HK, Min CK, Lee S, Chung YJ, Yoo NJ and Lee SH: Somatic mutations of JAK1 and JAK3 in acute leukemias and solid cancers. Clin Cancer Res 14: 3716-3721, 2008.

12. You Z, Xu D, Ji J, Guo W, Zhu W and He J: JAK/STAT signal pathway activation promotes progression and survival of human oesophageal squamous cell carcinoma. Clin Transl Oncol 14: 143-149, 2012.

13. Liu D, Huang Y, Zeng J, Chen B, Huang N, Guo N, Liu L, Xu H, Mo X and Li W: Down-regulation of JAK1 by RNA interference inhibits growth of the lung cancer cell line A549 and interferes with the PI3K/mTOR pathway. J Cancer Res Clin Oncol 137: 1629-1640, 2011.

14. Hirsch FR, Varella-Garcia M, McCoy J, West H, Xavier AC, Gumerlock P, Bunn PA Jr, Franklin WA, Crowley J and Gandara DR; Southwest Oncology Group: Increased epidermal growth factor receptor gene copy number detected by fluorescence in situ hybridization associates with increased sensitivity to gefitinib in patients with bronchioloalveolar carcinoma subtypes: A southwest oncology group study. J Clin Oncol 23: 6838-6845, 2005.

15. Lee Y, Shim HS, Park MS, Kim JH, Ha SJ, Kim SH and Cho BC: High EGFR gene copy number and skin rash as predictive markers for EGFR tyrosine kinase inhibitors in patients with advanced squamous cell lung carcinoma. Clin Cancer Res 18: 1760-1768, 2012.

16. Shan L, Wang Z, Guo L, Sun H, Qiu T, Ling Y, Li W, Li L, Liu X, Zheng B, et al: Concurrence of EGFR amplification and sensitizing mutations indicate a better survival benefit from EGFR-TKI therapy in lung adenocarcinoma patients. Lung Cancer 89: 337-342, 2015.

17. Koh Y, Jang B, Jeon YK, Kim TM, Lee SH, Kim DW, Chung DH, Kim YT, Kim YW and Heo DS: EGFR gene copy number gain is related to high tumor SUV and frequent relapse after adjuvant chemotherapy in resected lung adenocarcinoma. Jpn J Clin Oncol 41: 548-554, 2011.

18. UICC and IUAC: TNM classification of malignant tumours. Wiley, New York, NY, 2002.

19. Travis W, Brambilla E, Muller-Hermlink H and Harris C: World Health Organization classification of tumours. Pathology and genetics of tumours of the lung, pleura, thymus and heart. IARC Press, Lyon, 2004. 
20. NCCN: NCCN Clinical practice guideline in oncology-non-small cell lung cancer guideline 2004. http://www.nccn.org. 2004.

21. Dacic S, Flanagan M, Cieply K, Ramalingam S, Luketich J, Belani C and Yousem SA: Significance of EGFR protein expression and gene amplification in non-small cell lung carcinoma. Am J Clin Pathol 125: 860-865, 2006.

22. Varella-Garcia M: Stratification of non-small cell lung cancer patients for therapy with epidermal growth factor receptor inhibitors: The EGFR fluorescence in situ hybridization assay. Diagn Pathol 1: 19, 2006.

23. Murakami T, Takigawa N, Ninomiya T, Ochi N, Yasugi M, Honda Y, Kubo T, Ichihara E, Hotta K, Tanimoto M and Kiura K: Effect of AZD1480 in an epidermal growth factor receptor-driven lung cancer model. Lung Cancer 83: 30-36, 2014.

24. Hedvat M, Huszar D, Herrmann A, Gozgit JM, Schroeder A, Sheehy A, Buettner R, Proia D, Kowolik CM, Xin H, et al: The JAK2 inhibitor AZD1480 potently blocks Stat3 signaling and oncogenesis in solid tumors. Cancer Cell 16: 487-497, 2009.
25. Torres AF, Nogueira C, Magalhaes J, Costa IS, Aragao A, Gomes Neto A, Martins F and Tavora F: Expression of EGFR and molecules downstream to PI3K/Akt, Raf-1-MEK-1-MAP (Erk1/2), and JAK (STAT3) pathways in invasive lung adenocarcinomas resected at a single institution. Anal Cell Pathol (Amst) 2014: 352925,2014

26. Cordero JB, Stefanatos RK, Myant K, Vidal M and Sansom OJ: Non-autonomous crosstalk between the Jak/Stat and Egfr pathways mediates Apc1-driven intestinal stem cell hyperplasia in the Drosophila adult midgut. Development 139: 4524-4535, 2012.

27. Huang Y, Li X, Jiang J and Frank SJ: Prolactin modulates phosphorylation, signaling and trafficking of epidermal growth factor receptor in human T47D breast cancer cells. Oncogene 25: 7565-7576, 2006. 